\title{
RECURSIVELY CATEGORICAL LINEAR ORDERINGS
}

\author{
J. B. REMMEL ${ }^{1}$
}

\begin{abstract}
A recursive structure $\mathscr{Q}$ is recursively categorical if every recursive structure $\mathbb{Q}^{\prime}$ isomorphic to $\mathbb{Q}$ is recursively isomorphic to $\mathscr{Q}$. We classify the recursively categorical linear orderings as precisely those recursive linear orderings $L$ which have only finitely many elements with an immediate successor.
\end{abstract}

A structure $Q$ over a recursive language $\mathcal{L}$ is said to be recursive if $A$, the domain of $Q$, is recursive and there is a uniform effective procedure for deciding whether $\mathbb{Q}$ satisfies any atomic formula $\varphi\left(a_{0}, \ldots, a_{n}\right)$ with $a_{0}, \ldots, a_{n} \in A$. Two recursive structures $Q$ and $Q^{\prime}$ are recursively isomorphic, denoted $\mathbb{Q} \approx_{r} \mathbb{Q}^{\prime}$, if there is a partial recursive isomorphism $f$ from $Q$ onto $Q^{\prime}$. We say a recursive structure $Q$ is recursively categorical if any recursive structure $Q^{\prime}$ isomorphic to $Q$ is recursively isomorphic to $\mathbb{Q}$. The notion of recursive categoricity was first defined for groups by Mal'cev [3] and is referred to in the Soviet literature as autostability. In recent years, the notion of recursive categoricity has been studied widely in the literature. General semantic conditions for when decidable models are recursively categorical was given by Nurtazin [4] and similar results were found by Ash and Nerode for models in which one can effectively decide all $\Sigma_{1}$-formulas. The recursively categorical structures for various theories have been classified, including: Boolean algebras, independently by Goncharov [2] and La Roche [5] (the first full proof in the literature is found in Remmel [7]); Boolean algebras with additional predicates for atoms or atoms and atomless elements by Remmel [8], [9]; Abelian $p$-groups by Smith [10]; and decidable dense two-dimensional partial orderings by Remmel and Manaster [4]. The main result of this paper is the classification of the recursively categorical recursive linear orderings as precisely those recursive linear orderings $L$ which have only finitely many elements which have immediate successors. We end the paper with a brief discussion of the relationship between our classification and the known classification of recursively categorical Boolean algebras. Finally we should note that Theorem 1 to follow does not follow from the general work of Nurtazin or Ash and Nerode since Goncharov [2] has constructed recursive linear orderings $K$ such that one cannot effectively decide the $\Sigma_{1}$-formulas in any recursive linear ordering isomorphic to $K$.

Given a linear ordering $L=\left\langle A,<_{L}\right\rangle$, we say the pair $(x, y)$ is a successivity of $L$, written $x \rightarrow_{L} y$, if $x<_{L} y$ and there is no $z \in A$ such that $x<_{L} z<_{L} y$. We let

Received by the editors September 29, 1980.

1980 Mathematics Subject Classification. Primary 02F27; Secondary 06A05.

${ }^{1}$ Partially supported by NSF Grant MCS79-03406. 
$\varphi_{0}, \varphi_{1}, \ldots$ be an effective list of all 1:1 partial recursive functions. We write $\varphi_{e}^{s}(x) \downarrow$ if the effective procedure for calculating $\varphi_{e}(x)$ gives an output in $s$ or fewer steps. We write $\varphi_{e}(x) \downarrow$ if $\exists s\left(\varphi_{e}^{s}(x)\right) \downarrow .\langle$,$\rangle will denote a 1: 1$ recursive pairing function from $N \times N$ onto $N$ where $N$ is the natural numbers. $\eta$ and $\omega$ will denote the order types of the rationals and $N$, respectively. For $n \in \omega, \bar{n}$ will denote the linear ordering of $0, \ldots, n-1$ under the usual ordering.

THEOREM 1. A recursive linear order $L$ is recursively categorical iff $L$ has only finitely many successivities.

Proof. The "if" part of the theorem is simple. First note that Cantor's basic back and forth argument, which shows that any two dense linear orderings without endpoints are isomorphic, is effective. Thus any two recursive linear orderings isomorphic to $\eta$ are recursively isomorphic. Now if $L$ has only finitely many successivities, then it is easy to see that $L$ is isomorphic to a finite sum $\sum_{i-1}^{n} L_{i}$ where each $L_{i}$ is either finite or isomorphic to $\eta$. Then if $L^{\prime} \approx L$ and $L^{\prime}$ is recursive, we need only a finite amount of information to decompose $L=\sum_{i=1}^{n} L_{i}$ and $L^{\prime}=\sum_{i=1}^{n} L_{i}^{\prime}$ into finite sums where for each $i, L_{i} \approx L_{i}^{\prime}, L_{i}$ and $L_{i}^{\prime}$ are recursive, and $L_{i}$ and $L_{i}^{\prime}$ are either finite or isomorphic to $\eta$. It thus easily follows that $L \approx_{r} L^{\prime}$.

For the "only if" part of the theorem suppose that $L$ is a recursive linear order with infinitely many successivities. We shall construct a recursive linear ordering $R$ in stages which is isomorphic to $L$ but which is not recursively isomorphic to $L$. We can assume without loss of generality that the domain of $L$ is $N$ and we will construct $R$ to have domain $N$. At each stage $s$ of our construction we shall specify the linear order $<_{R}$ on $\{0, \ldots, s\}$ and a finite isomorphism $f_{s}:\{0, \ldots, s\} \rightarrow$ $\{0, \ldots, s\}$ such that for all $i<_{j} \leqslant s, i<_{L} j$ iff $f_{s}(i)<_{R} f_{s}(j)$. For notational convenience, we let $l_{0}^{s}, \ldots, l_{s}^{s}$ be the elements $\{0, \ldots, s\}$ in increasing $<_{L}$ order and $r_{0}^{s}, \ldots, r_{s}^{s}$ be the elements $\{0, \ldots, s\}$ in increasing $<_{R}$ order. Thus for all $j \leqslant s, f_{s}\left(l_{j}^{s}\right)=r_{j}^{s}$. It will be the case that for certain $x$ and $s$ that $f_{s}(x) \neq f_{s+1}(x)$, but we will ensure that for all $x, \lim _{s} f_{s}(x)=f(x)$ exists and that $f$ is an isomorphism from $L=\left\langle N,<_{L}\right\rangle$ onto $R=\left\langle N,<_{R}\right\rangle$.

The basic idea of our construction is to use the infinitely many successivities of $L$ to diagonalize over all possible recursive isomorphisms. Thus our construction will ensure that we meet the following set of requirements for $e=0,1,2, \ldots$.

$\mathrm{P}_{e}: \quad$ If $\varphi_{e}$ is an order preserving map from $L$ into $R$, then there exist $a, b \in L$ with $a \rightarrow_{L} b$ such that $\varphi_{e}(a) \nrightarrow_{R} \varphi_{e}(b)$.

The basic action to meet the requirement $\mathrm{P}_{e}$ is as follows. Suppose at stage $s$, we have $l_{i}^{s} \rightarrow_{L} l_{i+1}^{s}, \varphi_{e}^{s}\left(l_{i}^{s}\right) \downarrow, \varphi_{e}^{s}\left(l_{i+1}^{s}\right) \downarrow$, and for some $j<s, \varphi_{e}\left(l_{i}^{s}\right)=r_{j}^{s}$ and $\varphi_{e}\left(l_{i+1}^{s}\right)=$ $r_{j+1}^{s}$. Then at stage $s+1$, we will define $<_{R}$ so that $r_{j}^{s}<_{R} s+1<_{R} r_{j+1}^{s}$, thus ensuring that $r_{j}^{s} \nrightarrow_{R} r_{j+1}^{s}$ and requirement $\mathrm{P}_{e}$ is met. There are several conflicts that we must overcome in order to carry out this basic strategy. First, since it is not the case that $l_{j}^{s}<_{L} s+1<_{L} l_{j+1}^{s}$, we will be forced to have $f_{s}(x) \neq f_{s+1}(x)$ for some $x \leqslant s$ when we put $r_{j}^{s}<_{R} s+1<_{R} r_{j+1}^{s}$. So to ensure that $\lim _{s} f_{s}(x)$ exists for all $x$ and that $f$ is onto, we will allow such an action to be taken for requirements $\mathbf{P}_{e}$ iff 
we can arrange to have $f_{s}(x)=f_{s+1}(x)$ for all $x \in\{0, \ldots, e\} \cup f_{s}^{-1}(\{0, \ldots, e\})$. Another problem is that we cannot effectively tell when $a \rightarrow_{L} b$. Indeed, Goncharov [2] has constructed a recursive linear ordering $K$ such that there is no recursive linear ordering $K^{\prime} \approx K$ such that the successivities of $K^{\prime}$ are recursive. However the complement of the successivities of $L$,

$$
C=\left\{\langle x, y\rangle \mid x \Varangle_{L} y \text { or } \exists u\left(x<_{L} u<_{L} y\right)\right\},
$$

is an r.e. set. We let

$$
C^{s}=\left\{\langle x, y\rangle \mid x, y \leqslant s \& x \Varangle_{L} y \text { or } \exists u \leqslant s\left(x<_{L} u<_{L} y\right)\right\} .
$$

The $\langle x, y\rangle \notin C^{s}$ approximate successitivites of $L$ at stage $s$ and we use such $\langle x, y\rangle$ to carry out our strategy for $\mathrm{P}_{e}$. Of course we may later find that $\langle x, y\rangle \in C$, in which case we will have to take additional action for $\mathrm{P}_{\boldsymbol{e}}$. Nevertheless, our construction will ensure that eventually we will take an action for $\mathrm{P}_{e}$ as above using some $\left\langle l_{i}^{s}, l_{i+1}^{s}\right\rangle$ such that $\left\langle l_{i}^{s}, l_{i+1}^{s}\right\rangle \notin C$. We let $c_{0}^{s}\left\langle c_{1}^{s}<\cdots\right.$ be a list of $N-C^{s}$ in order of magnitude.

Construction.

Stage 0 . Let $f_{0}(0)=0$.

Stage $s+1$. Look for the least $n=n(s+1) \leqslant s+1$ such that:

(i) $c_{n}^{s+1}=\left\langle l_{i}^{s}, l_{i+1}^{s}\right\rangle$ for some $i \leqslant s$,

(ii) $n=\langle e, d\rangle$ and for all $x, y \leqslant s$ if $\varphi_{e}^{s+1}(x) \downarrow, \varphi_{e}^{s+1}(y) \downarrow$ and $\varphi_{e}(x), \varphi_{e}(y) \leqslant s$, then $x<_{L} y$ iff $\varphi_{e}(x)<_{R} \varphi_{e}(y)$,

(iii) $\varphi_{e}^{s+1}\left(l_{i}^{s}\right) \downarrow, \varphi_{e}^{s+1}\left(l_{i+1}^{s}\right) \downarrow, \varphi_{e}\left(l_{i}^{s}\right)=r_{j}^{s}$, and $\varphi_{e}\left(l_{i+1}^{s}\right)=r_{j+1}^{s}$ for some $j<s$.

(iv) There exist $u, v \in\{0, \ldots, s\} \cup\{-\infty,+\infty\}$ such that $l_{u}^{s} \leqslant_{L} l_{j}^{s}<_{L} l_{j+1}^{s} \leqslant{ }_{L} l_{v}^{s}$ and $l_{u}^{s}<_{L} s+1<_{L} l_{v}^{s}$ and there is no $x \in\{0, \ldots, e\} \cup f_{s}^{-1}(\{0, \ldots, e\})$ with $l_{u}^{s}<_{L} x<_{L} l_{v}^{s}$. $\left(u=-\infty\right.$ means $s+1<_{L} l_{0}^{s}$ and there is no $x$ as above with $x<l_{v}^{s}$, and $v=+\infty$ means $l_{s}^{s}<_{L} s+1$ and there is no $x$ as above with $l_{u}^{s}<x$.)

(v) If $k\left\langle n, k=\langle e, d\rangle\right.$, and $c_{k}^{s+1}=\left\langle l_{a}^{s}, l_{a+1}^{s}\right\rangle$ for some $a<s$, then $\varphi_{e}^{s+1}\left(l_{a}^{s}\right) \downarrow$, $\varphi_{e}^{s+1}\left(l_{a+1}^{s}\right) \downarrow, \varphi_{e}\left(l_{a}^{s}\right)=r_{p}^{s}$, and $\varphi_{e}\left(l_{a+1}^{s}\right)=r_{p+1}^{s}$ for some $p<s$ (obviously for such $k$, (iv) fails; for example it may be that $\left.l_{a}^{s}, l_{a+1}^{s+1} \in\{0, \ldots, e\}\right)$.

If there is no such $n$, we let $s+1<_{R} r_{0}^{s}$ if $s+1<_{L} l_{0}^{s}, r_{s}^{s}<_{R} s+1$ if $l_{s}^{s}<_{L}$ $s+1$, and $r_{m}^{s}<_{R} s+1<_{R} r_{m+1}^{s}$ if $l_{m}^{s}<_{L} s+1<_{L} l_{m+1}^{s}$ for some $m<s$. Then we define $f_{s+1}(s+1)=s+1$ and $f_{s+1}(x)=f_{s}(x)$ for all $x<s$. If there is such an $n$, assume that the $u$ and $v$ of (iv) are chosen so that $\langle u, v\rangle$ is as small as possible. We let $r_{j}^{s}<_{R} s+1<_{R} r_{j}^{s+1}$. By (iii), it is clear that since $f_{s+1}$ is defined to be the unique order isomorphism between $\left\langle\{0, \ldots, s+1\},\left\langle_{L}\right\rangle\right.$ and $\left\langle\{0, \ldots, s+1\},\left\langle_{R}\right\rangle\right.$, we have $f_{s+1}\left(l_{i}^{s}\right)=r_{i}^{s}$ if either $i<u$ or $v<i$ so that $f_{s+1}(x)=f_{s}(x)$ for all $x \in\{0, \ldots, e\} \cup f_{s}^{-1}(\{0, \ldots, e\})$.

This completes the description of the stages. It is clear that each stage is completely effective so that $R=\left\langle N,<_{R}\right\rangle$ is a recursive linear ordering. Thus to complete our proof we need only check that $f=\lim _{s} f_{s}$ is an isomorphism from $L$ onto $R$ and that all the requirements $P_{e}$ are satisfied. We prove both these facts by a simultaneous induction. Note, to prove that $f$ is an isomorphism, we need only check that for all $x, \lim _{s} f_{s}(x)$ and $\lim _{s} f_{s}^{-1}(x)$ exist, since at each stage $f_{s}$ is order preserving. Since $f_{s+1}(x) \neq f_{s}(x)$ or $f_{s+1}^{-1}(x) \neq f_{s}^{-1}(x)$ only if the $n(s+1)$ chosen at 
stage $s+1$ is of the form $\langle e, q\rangle$ where $e<x$, all we need to do to prove that $\lim _{s} f_{s}(x)$ and $\lim _{s} f_{s}^{-1}(x)$ exist for all $x$ is to show that for each $e$ there are only finitely many stages $s$ where $n(s)$ is of the form $\langle e, q\rangle$. So assume by induction that all the requirements $\mathrm{P}_{0}, \ldots, \mathrm{P}_{e-1}$ are met and that there is a stage $s_{0}$ large enough so that for all $s \geqslant s_{0}$, the $n(s)$ chosen at stage $s$, if any, is of the form $\langle k, d\rangle$ where $k \geqslant e$. First we can assume that $\varphi_{e}$ is an isomorphism from $\left\langle\right.$ domain of $\left.\varphi_{e},<_{L}\right\rangle$ onto $\left\langle\right.$ range of $\left.\varphi_{e},<_{R}\right\rangle$. For otherwise requirement $\mathbf{P}_{e}$ is met and there will be a stage $s>s_{0}$ such that there is $x, y \leqslant s$ in the domain of $\varphi_{e}^{s}$ such that $x<_{L} y$ but $\varphi_{e}(x) \Varangle_{R} \varphi_{e}(y)$ and $\varphi_{e}(x), \varphi_{e}(y) \leqslant s$. Then by condition (ii) of our definition of $n(s)$ we know that $n(t)$ is not of the form $\langle e, q\rangle$ for $t \geqslant s$.

Next let $c_{0}, c_{1}, \ldots$ be a list of $C$ in order of magnitude and $n_{0}<n_{1}<\ldots$ be a list of those $n$ 's such that $n=\langle e, q\rangle$ for some $q$. Now if there is some $k$ such that $c_{n_{k}}=\langle x, y\rangle$ and either not both $\varphi_{e}(x)$ and $\varphi_{e}(y)$ are defined or $\varphi_{e}(x)$ and $\varphi_{e}(y)$ are defined but $\varphi_{e}(x) \nrightarrow_{R} \varphi_{e}(y)$, then clearly requirement $\mathrm{P}_{e}$ is met. Moreover, suppose $s_{1}>s_{0}$ is large enough so that $c_{i}^{s_{1}}=c_{i}$ for all $i \leqslant n_{k}$, and if both $\varphi_{e}(x)$ and $\varphi_{e}(y)$ are defined then $x, y, \varphi_{e}(x), \varphi_{e}(y) \leqslant s_{1}, \varphi_{e}^{s_{1}}(x) \downarrow, \varphi_{e}^{s_{1}}(y) \downarrow$, and $\exists u \leqslant s_{1}\left(\varphi_{e}(x)<_{R} u\right.$ $\left.<_{R} \varphi_{e}(y)\right)$. Now it is easy to see that if $\left.s\right\rangle s_{1}$ and $n(s)=\langle e, q\rangle$, then condition (v) forces $n(s)<n_{k}$. Similarly if $t>s$ and the $n(t)=\left\langle e, q^{\prime}\right\rangle$, then $n(t)<n(s)$, etc. Thus there can only be finitely many stages $s>s_{1}$ where $n(s)$ is of the form $\langle e, q\rangle$.

Finally we are reduced to the case where for all $n_{j}$, if $c_{n_{j}}=\left\langle x_{j}, y_{j}\right\rangle$, then both $\varphi_{e}\left(x_{j}\right)$ and $\varphi_{e}\left(y_{j}\right)$ are defined and $\varphi_{e}\left(x_{j}\right) \rightarrow_{R} \varphi_{e}\left(y_{j}\right)$. We claim that this case never happens. For let $a_{0}<_{L} a_{1}<_{L} \cdots<_{L} a_{m}$ be the l-ordering of the elements $\{0, \ldots, e\} \cup f_{s_{0}}^{-1}(\{0, \ldots, e\})$ and $b_{0}<_{R} b_{1}<_{R} \cdots<_{R} b_{m}$ be the $R$-ordering of their corresponding images under $f_{s_{0}}$. By our assumptions on $\varphi_{e}$ we know that $\left\langle\varphi_{e}\left(x_{0}\right), \varphi_{e}\left(y_{0}\right)\right\rangle,\left\langle\varphi_{e}\left(x_{1}\right), \varphi_{e}\left(y_{1}\right)\right\rangle, \ldots$ is a list of infinitely many distinct $R$-successivities. It follows from the fact that $f^{s}$ is always a finite isomorphism, that for some $k, \varphi_{e}\left(x_{k}\right)$ and $\varphi_{e}\left(y_{k}\right)$ lie in one of the intervals $\left(-\infty, b_{0}\right], \ldots,\left[b_{i}, b_{i+1}\right], \ldots,\left[b_{m},+\infty\right)$ where the corresponding interval in $L$ is infinite. Say $b_{i}<_{R} \varphi_{e}\left(x_{k}\right)<\varphi_{e}\left(y_{k}\right)<b_{i+1}$ where $\left[a_{i}, a_{i+1}\right]$ is infinite in $L$. It is now easy to see that there must be a stage $s>s_{0}$ large enough so that for all $i \leqslant n_{k}$ : (a) $c_{i}^{s}=c_{i}$; (b) $x_{i}, y_{i}, \varphi_{e}\left(x_{i}\right)$ and $\varphi_{e}\left(y_{i}\right) \leqslant s$; and (c) $\varphi_{e}^{s}\left(x_{i}\right) \downarrow$ and $\varphi_{e}^{s}\left(y_{i}\right) \downarrow$ and, moreover, $a_{i}<_{L} s+1<_{L} a_{i+1}$. It then follows that $n_{k}$ satisfies the conditions to be $n(s+1)$. By choice of $s>s_{0}, n(s+1)$ is defined and $n(s+1)=n_{i}$ for some $i \leqslant k$ so that $n(s+1)$ is of the form $\langle e, q\rangle$. But then our actions taken at stage $s+1$ ensure that $\varphi_{e}\left(x_{i}\right) \nrightarrow_{R} \varphi_{e}\left(y_{i}\right)$, contradicting our assumptions on $\varphi_{e}$.

We note that given any effective sequence $L_{0}, L_{1}, \ldots$ of recursive linear orderings with infinitely many successivities, one can easily interweave the same type of requirements as in Theorem 1 to construct a recursive linear ordering $L^{\prime}$ isomorphic to $L_{0}$ but where $L \mathscr{\star}_{r} L_{i}$ for any $i$. Thus the following holds.

COROLlaRY 1. Let $L$ be a recursive linear ordering. Then

(a) The classical isomorphism type of $L, \Gamma(L)$, contains either one or infinitely many recursive isomorphism types.

(b) If $L$ has infinitely many successivities, there is no effective list $L_{0}, L_{1}, \ldots$ of recursive linear orderings with infinitely many successivities which contain at least one representative in every recursive isomorphism type in $\Gamma(L)$. 
Given a recursive linear order $L$ with a first element, the set of all left-closed right-open intervals of $L$ forms a recursive Boolean algebra, $B_{L}$, called the interval algebra of $L$. Goncharov and La Roche showed that the recursively categorical Boolean algebras are the ones with finitely many atoms. Thus a recursive linear ordering $L$ with a first element is recursively categorical iff $B_{L}$ is recursively categorical. We do not know whether Goncharov and La Roche's result follows from Theorem 1 or vice versa. Indeed there is some evidence to suggest that this is not the case. The problem is that there are many nonisomorphic linear orderings which give rise to the same Boolean algebra. In fact, if we add additional predicates, the recursive categoricity property does not transfer to the interval algebra. For example, we showed in [8] that if we add an additional predicate $A(x)$ for " $x$ is an atom" (so that we consider only recursive Boolean algebras with recursive sets of atoms) then the recursively categorical Boolean algebras with recursive sets of atoms is the class $D$ which contains all finite Boolean algebras, the atomless Boolean algebra, and the Boolean algebra of finite and cofinite subsets of $N$ and is closed under finite products. The corresponding additional predicate for linear orderings is the binary predicate $S(x, y)$ for " $x \rightarrow y$ ". Now $\tilde{C}$, the Boolean algebra generated by all the closed intervals of the rationals $\eta$ is a recursive Boolean algebra with a recursive set of atoms and is isomorphic to the interval algebras on $\overline{1}+(\eta \times \omega)$ and $\overline{1}+(\eta \times \bar{k})$ for any $k \geqslant 2$ where $\bar{k}$ is the discrete linear ordering on $k$ elements. But $\tilde{C}$ is not recursively categorical in the class of recursive Boolean algebras with recursive sets of atoms while it is easy to show that $\overline{1}+(\eta \times \bar{k})$ is recursively categorical but $\overline{1}+(\eta \times \omega)$ is not recursively categorical in the class of recursive linear orderings with recursive successivities. We note that the classification of the recursively categorical linear orderings with recursive successivities is open.

\section{REFERENCES}

1. C. J. Ash and A. Nerode, Intrinsically recursive relation, Effective Aspects of Algebra (J. N. Crossley, ed.), Upsidedown A Book Co., Clayton-Victoria, Australia, 1980.

2. S. S. Goncharov, Some properties of the constructivization of Boolean algebras, Sibirsk Mat. Ż. 16 (1975), 264-278.

3. A. I. Mal'cev, On recursive abelian groups, Soviet Math. 3 (1962), 1431-1432.

4. A. B. Manaster and J. B. Remmel, Recursively categorical decidable dense two dimensional partial orderings (to appear).

5. P. E. LaRoche, Recursively presented Boolean algebras, Notices Amer. Math. Soc. 24 (1977), A-552.

6. A. T. Nurtazin, Strong and weak constructivizations and computable families, Algebra and Logic 13 (1975), 177-184.

7. J. B. Remmel, Recursive isomorphisms of recursive Boolean algebras, J. Symbolic Logic (to appear).

8. _ Recursive Boolean algebras with recursive sets of atoms, J. Symbolic Logic (to appear).

9. __ Recursive isomorphisms of recursive Boolean algebras and atomic elements (in preparation).

10. R. L. Smith, Two theorems on autostability in p-groups (Proc. Conf. Math. Logic, Univ. Connecticut), Lecture Notes in Math. (to appear).

Department of Mathematics, University of California, San Diego, la Jolla, California 92093 